\title{
Strong Einstein-Podolsky-Rosen steering with unconditional entangled states
}

\author{
Sebastian Steinlechner, Jöran Bauchrowitz, Tobias Eberle, and Roman Schnabel \\ Institut für Gravitationsphysik, Leibniz Universität Hannover, and Max-Planck-Institut für Gravitationsphysik (Albert-Einstein-Institut), \\ Callinstraße 38, 30167 Hannover, Germany
}

(Received 30 November 2011; revised manuscript received 9 November 2012; published 6 February 2013)

\begin{abstract}
In 1935 Schrödinger introduced the terms entanglement and steering in the context of the famous gedanken experiment discussed by Einstein, Podolsky, and Rosen (EPR). Here, we report on a sixfold increase of the observed EPR-steering effect with regard to previous experiments, as quantified by the Reid criterion. We achieved an unprecedented low conditional variance product of about $0.04<1$, where 1 is the upper bound below which steering is demonstrated. The steering effect was observed on an unconditional two-mode-squeezed entangled state that contained a total vacuum state contribution of less than $8 \%$, including detection imperfections. Together with the achieved high interference contrast between the entangled state and a bright coherent laser field, our state is compatible with efficient applications in high-power laser interferometers and fiber-based networks for entanglement distribution.
\end{abstract}

DOI: 10.1103/PhysRevA.87.022104

PACS number(s): 03.65.Ud, 03.67.Bg, 42.50.Dv, 42.50.Xa

\section{INTRODUCTION}

Ever since the landmark article by Einstein, Podolsky, and Rosen (EPR) [1] was published, entanglement has been demonstrated in a large variety of physical systems [2-7]. Recent theoretical and experimental work in quantum information [8-10] has sparked an interest in EPR steering. The term itself was already coined by Schrödinger in 1935 [11] in response to the original EPR gedanken experiment.

The steering effect can be described as follows. One party, Alice, repeatedly sends a defined physical system to another party, Bob. She then proves to Bob that she can predict his measurement outcomes on this system with more precision than would be possible for any separable (classical) state. In the actual case of a shared steering state, Bob would find rather broad distributions of his measurement results for two noncommuting observables. When he evaluates the discrepancies between Alice's predictions and his measurement results, however, Bob realizes that the accordingly mutual uncertainties are less than allowed by a pure separable minimum uncertainty state, as quantified by Heisenberg's uncertainty principle. It therefore seems that Alice accomplishes a phase-space steering of the measurement outcomes at Bob's side merely by performing measurements on her subsystem. Of course, in contrast to what the term steering might suggest, in the course of Alice's measurements no information is exchanged with Bob's subsystem. But when she sends her predictions, Bob realizes that her knowledge exceeds the fundamental limit for a separable state and its noncommuting observables, such as position and momentum.

EPR as well as Schrödinger considered the example of an idealized, pure bipartite entangled state of continuous variables (CVs). In 1989, Reid proposed a criterion for the EPR entanglement of nonidealized Gaussian, continuous-variable systems, i.e., for decohered, mixed Gaussian states [12]. More recently, Cavalcanti et al. $[13,14]$ derived criteria for nonidealized discrete systems, and Wiseman and co-workers developed a general theory of experimental EPR-steering criteria applicable to discrete as well as continuous-variable observables [8,9]. It was shown that the Reid criterion can be rederived within this formalism. The first demonstration of
EPR steering was achieved by Ou et al. in 1992, followed by several other experiments in the CV regime [15-23]. In 2003, Bowen et al. experimentally demonstrated in the Gaussian regime that EPR entanglement is indeed more demanding than just establishing entanglement [17]. In 2004, Howell demonstrated the EPR paradox for continuous variables of single photons using postselection [24]. A review on these experiments is given in [25]. EPR steering was also observed in several experiments with discrete variables, based on photon counting and postselection [10,26-28]. Recently, we have shown that the intrinsic asymmetry in the steering scenario can lead to one-way steering, where only Alice can steer Bob but not vice versa [29].

Several quantum-information protocols for unconditional $\mathrm{CV}$ entanglement are known. In each of these, quantitatively strong nonclassical properties are required for useful implementations. For example, dense coding can increase the capacity of a quantum-information channel, but requires more than $4.8 \mathrm{~dB}$ of two-mode squeezing to surpass classical schemes [30]. Analogously, the obtained secure bit rate in $\mathrm{CV}$ quantum key distribution depends on the strength of the entanglement [31]. In the field of quantum metrology, recent theoretical and experimental works show promising sensitivity improvements with unconditional entangled states [32-35].

Here we report on the continuous observation of strong unconditional EPR steering in the Gaussian regime using twomode squeezed states. The EPR steering strength is quantified to $\mathcal{E}^{2}=0.041 \pm 0.005<1$, where unity is the benchmark below which the steering effect is demonstrated [9]. This value is, to the best of our knowledge, the strongest unconditional EPR entanglement measured to date and a more than sixfold improvement over previous values [22]. The improvement was made possible by the recent advances in squeezed light sources [36,37], which allowed us to stably operate two such sources simultaneously with detected squeezing values of about $10 \mathrm{~dB}$. A special property of our EPR state is its low vacuum state contribution of just $8 \%$. The EPR entanglement is contained in a well-defined $\mathrm{TEM}_{00}$ mode and is therefore ideally suited for possible applications in optical networks and high-power laser interferometers. 


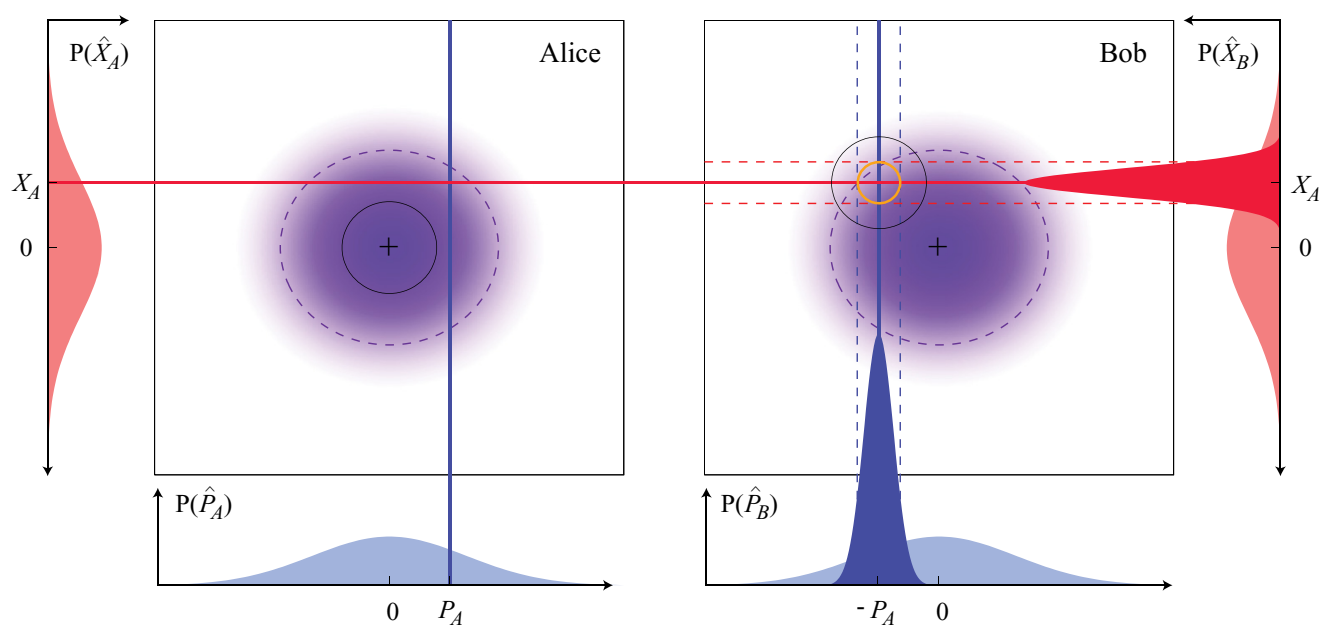

FIG. 1. (Color online) Graphical representation of the EPR-Reid criterion as a measure for the CV EPR-steering task. Alice and Bob share an entangled state with probability distributions for each quadrature as given by the four broad projections for $\hat{X}$ and $\hat{P}$ in red and blue, respectively. From a measurement result $X_{A}$ (solid red horizontal line) at her side, Alice can predict the measurement outcome at Bob's detector with an uncertainty as given by the dashed red horizontal lines. The same applies to a measurement of $P_{A}$, as indicated by the blue vertical lines. The two narrow Gaussian projections and thus the area enclosed by the small orange circle are measures for Alice's ability to predict Bob's measurement results. For our experimental results, which are drawn here to scale, this conditional uncertainty circle has about one fifth the variance of the vacuum (large black circle), which would be the lower uncertainty limit had Alice and Bob not shared an entangled state.

To qualify and quantify the EPR steering, we use the EPRReid criterion [12] for the field quadratures $\hat{X}$ and $\hat{P}$,

$$
\mathcal{E}_{B \mid A}^{2}=\Delta_{B \mid A}^{2} \hat{X} \Delta_{B \mid A}^{2} \hat{P} \stackrel{!}{<} 1,
$$

where $\Delta_{B \mid A}^{2} \hat{O}=\min _{g_{O}} \Delta^{2}\left(\hat{O}_{B}-g_{O} \hat{O}_{A}\right)$ is the conditional variance and $\Delta^{2} \hat{O}$ denotes the variance of the observable $\hat{O}$. The goal for Alice is to minimize the mutual uncertainty, i.e., the conditional variances. If Alice is able to fulfill inequality (1), Bob will be convinced that they indeed share an entangled state. The critical value of unity in Eq. (1) comes from the fact that classical correlations between two beams can only be determined with at most the accuracy of the vacuum's zero-point fluctuations. Throughout this paper, we normalize the variance of this so-called vacuum noise to unity, $\Delta^{2} \hat{X}^{\text {vac }}=$ $\Delta^{2} \hat{P}^{\mathrm{vac}} \equiv 1$.

Figure 1 illustrates the EPR-Reid criterion used in this work. It shows the joint two-mode quasiprobability distribution of the EPR entangled state shared by Alice and Bob. The four broad Gaussian distributions give the measurement statistics for measuring one or the other noncommuting observable $\hat{X}$ and $\hat{P}$ on the respective subsystem $A$ or $B$. The figure further illustrates the success of our experiment drawn to scale. For both quadratures we achieved a conditional variance about five times smaller than the minimal uncertainty product possible for separable states. This is represented in the illustration by the small orange circle on the right, compared to the larger black circle.

\section{EXPERIMENT}

Figure 2 shows a schematic of our experimental setup. Two squeezed-light resonators (SLRs) produced amplitudequadrature squeezed-light fields at a wavelength of $1064 \mathrm{~nm}$. These were overlapped with a relative phase of $\pi / 2$ on a
50:50 beam splitter, thereby creating two-mode squeezing. The quadrature amplitudes $\hat{X}$ and $\hat{P}$ of the two output modes were detected with two balanced homodyne detectors at Alice's and Bob's sites. Both detector outputs were passively subtracted and then measured with a spectrum analyzer at a Fourier frequency of $5 \mathrm{MHz}$ with a resolution bandwidth of $300 \mathrm{kHz}$.

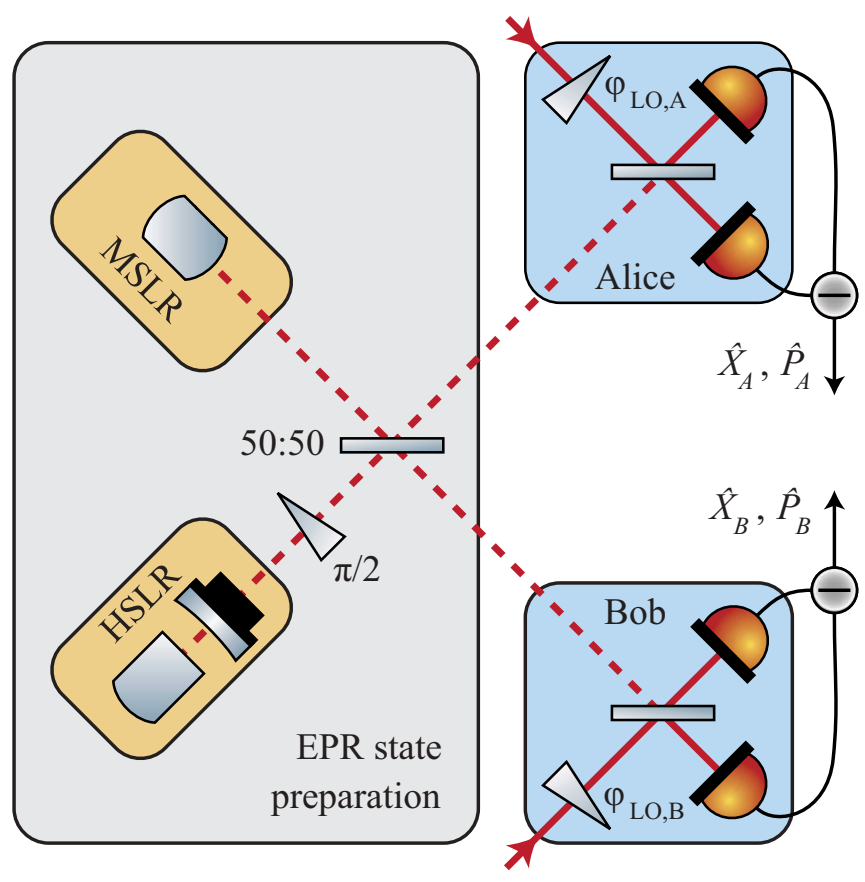

FIG. 2. (Color online) Schematic of the experimental setup. Two squeezed beams coming from a monolithic and a half-monolithic squeezed-light resonator, MSLR and HSLR, are overlapped at a 50:50 beam splitter, thereby producing a bipartite EPR entangled state. The measurements at Alice and Bob are performed by balanced homodyne detection. 
We used type I parametric down-conversion in periodically poled potassium titanyl phosphate (PPKTP) to generate the squeezed input fields. The two squeezed-light resonators differed in that one was monolithic (MSLR), with cavity mirror coatings directly applied to the crystal's curved end faces, while the other was half-monolithic (or hemilithic, HSLR), with the cavity between one crystal surface and a separate, piezoactuated mirror. In both cases, one crystal surface was highly reflecting at both the $1064 \mathrm{~nm}$ fundamental wavelength and the $532 \mathrm{~nm}$ pump field. The outcoupling mirrors each had a power reflectivity of $90 \%$ for the fundamental and $20 \%$ for the harmonic field. Peltier elements were thermally connected to both nonlinear crystals and used to temperature stabilize to the phase-matching condition. Most of the main laser light coming from a Nd:YAG nonplanar ring oscillator was converted into the pump field at $532 \mathrm{~nm}$ for the parametric down-conversion process in the SLRs. The second harmonic field was amplitude stabilized with a Mach-Zehnder type interferometer and spatially filtered in a mode-cleaning ring cavity. About $60 \mathrm{~mW}$ pump power was needed for each SLR. Submilliwatt control fields carrying radio-frequency phase modulations were injected into both squeezing resonators through their highly reflective mirror coatings. They were used to lock the monolithic as well as the hemilithic cavities on resonance by actuating the laser frequency and the piezodriven outcoupling mirror, respectively. The same phase modulations were also employed to control the phase of the pump field and to lock the homodyne detectors' quadrature angles. A small fraction of light was tapped off the main laser beam and spatially filtered. This beam was then divided to provide about $10 \mathrm{~mW}$ local oscillator power for each homodyne detector. The homodyne detectors where equipped with custom-made photodiodes with a quantum efficiency of $>99 \%$ and had a dark-noise clearance of about $22 \mathrm{~dB}$ below the vacuum noise.

\section{RESULTS}

A bipartite Gaussian entangled state is completely defined by its covariance matrix $\gamma$. Given the entries of $\gamma$, the EPRReid criterion (1) can be restated as [30]

$$
\begin{aligned}
\mathcal{E}_{B \mid A}^{2}= & \left(\Delta^{2} \hat{X}_{B}-\frac{\operatorname{Cov}\left(\hat{X}_{A}, \hat{X}_{B}\right)^{2}}{\Delta^{2} \hat{X}_{A}}\right) \\
& \times\left(\Delta^{2} \hat{P}_{B}-\frac{\operatorname{Cov}\left(\hat{P}_{A}, \hat{P}_{B}\right)^{2}}{\Delta^{2} \hat{P}_{A}}\right) .
\end{aligned}
$$

We performed six different measurements on our entangled system in order to partially reconstruct all relevant entries of its associated covariance matrix. These included the amplitude and phase quadratures at Alice and Bob, respectively, and the cross-correlations $\Delta^{2}\left(\hat{X}_{A}-\hat{X}_{B}\right)$ and $\Delta^{2}\left(\hat{P}_{A}+\hat{P}_{B}\right)$. From the latter we calculated the covariances between measurements at Alice and Bob via the identity

$$
\operatorname{Cov}\left(\hat{O}_{1}, \hat{O}_{2}\right)=\frac{1}{2}\left[\Delta^{2}\left(\hat{O}_{1}+\hat{O}_{2}\right)-\Delta^{2} \hat{O}_{1}-\Delta^{2} \hat{O}_{2}\right] .
$$

An example of the measured traces is given in Fig. 3. These clearly show the EPR-steering effect, $\mathcal{E}^{2}=0.042<1$, where we chose the scaling factors $g_{X}=1$ and $g_{P}=-1$. The same traces also show the inseparability of our system as expressed by the Duan criterion [38], $\Delta^{2}\left(\hat{X}_{A}-\hat{X}_{B}\right)+\Delta^{2}\left(\hat{P}_{A}+\hat{P}_{B}\right)<$ 4. From our measurements the left side evaluates to 0.41 , thus falling below the Duan threshold by almost a factor of 10 .

The partially reconstructed covariance matrix reads

$$
\gamma=\left(\begin{array}{cccc}
18.41 & (0) & 18.09 & (0) \\
(0) & 35.49 & (0) & -34.95 \\
18.09 & (0) & 17.98 & (0) \\
(0) & -34.95 & (0) & 34.61
\end{array}\right)
$$

Each entry has an associated relative error of about $5 \%$. The $\hat{P}$ quadratures show roughly twice the variance of the $\hat{X}$ quadratures, which can be attributed to almost $3 \mathrm{~dB}$ more antisqueezing produced in the monolithic squeezedlight resonator. The bracketed values where not measured but instead set to zero by our experimental arrangement, because the orientations of the squeezing ellipses as well as the local oscillator phases of the balanced homodyne detectors were precisely controlled in such a way that no correlations were introduced. In principle these values can also be measured independently, as we and others demonstrated before $[21,39,40]$. Such measurements, however, are rather involved or even introduce additional optical loss. Note that if the bracketed zeros did not correspond to the actual values, the generated entanglement strength would be underestimated but never overestimated.

Inserting the entries of the covariance matrix $\gamma$ into Eq. (2) yields $\mathcal{E}_{B \mid A}^{2}=0.039$ for the case where Alice steers the measurement outcome at Bob's detector. This result is slightly better than expected from Fig. 3, therefore we conclude that initially, Alice's scaling parameters were not perfectly chosen. The reverse setup, $\mathcal{E}_{A \mid B}^{2}$, performs similarly with an EPR value of 0.041 .

A comparison of $\gamma$ to a theoretical loss model yields an overall efficiency intrinsic to our physical system of $\xi=92 \%$. Internal loss of the squeezed-light resonators (about 2.5\%),

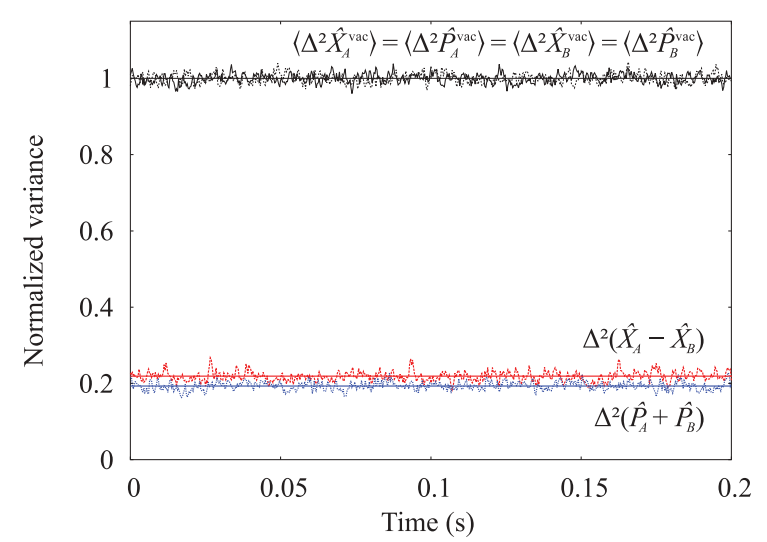

FIG. 3. (Color online) Variance of the sum and difference of the amplitude and phase quadratures at Alice and Bob. The traces were measured at a Fourier frequency of $5 \mathrm{MHz}$ and are normalized to the vacuum's zero-point fluctuations, $\Delta^{2} X^{\text {vac }}=\Delta^{2} P^{\text {vac }} \equiv 1$. Steering as characterized by the EPR-Reid criterion is clearly visible, here with scaling parameters $g_{X}=1$ and $g_{P}=-1$, i.e., $\Delta^{2}\left(\hat{X}_{A}-\hat{X}_{B}\right) \Delta^{2}\left(\hat{P}_{A}+\hat{P}_{B}\right)<1$. The traces were recorded with a resolution bandwidth of $300 \mathrm{kHz}$ and video bandwidth of $300 \mathrm{~Hz}$. Detection dark noise was $22 \mathrm{~dB}$ below the vacuum noise and was not subtracted from the measurement data. 
propagation loss $(1 \%)$, and imperfect mode overlap at the entanglement beam splitter (about $1.4 \%$ loss due to a fringe visibility of 0.993 ) lead to an EPR state preparation efficiency of $\eta=(95 \pm 1) \%$. We therefore obtain a homodyne detection efficiency of $\xi / \eta=(97 \pm 1) \%$, which is a reasonable value assuming a quantum efficiency of our custom-made photodiodes of $99 \%$, additional propagation loss of $0.6 \%$, and again a fringe visibility of about 0.993 .

\section{DISCUSSION AND CONCLUSION}

In this work we demonstrated unconditional EPR steering using continuous measurements of position- and momentumlike variables. We significantly improved the strength of the steering effect as quantified by Eq. (1) to $\mathcal{E}^{2}=0.04$. This value corresponds to an inseparability of $0.41<4$, according to Duan et al. [38].

Our improvement is closely linked to the recent advances in squeezed-light sources, which we successfully transferred and applied to our entanglement setup. The high quality of our state's spatial mode makes it also applicable in more complex optical networks, such as in interferometers, where an excellent mode matching is essential to achieving an overall high efficiency. From a fundamental point of view, the setting used in this work is able to asymptotically approach the original idealized gedanken experiment considered by Einstein, Podolsky, Rosen, and Schrödinger.

\section{ACKNOWLEDGMENTS}

We acknowledge support from the Centre for Quantum Engineering and Space-Time Research (QUEST), the International Max Planck Research School on Gravitational Wave Astronomy (IMPRS), the DFG Sonderforschungsbereich TR7, and the EU FP-7 project Q-ESSENCE, and helpful discussions with Jörg Duhme, Torsten Franz, Melanie Meinders, and Reinhard Werner.
[1] A. Einstein, B. Podolsky, and N. Rosen, Phys. Rev. 47, 777 (1935).

[2] A. Aspect, P. Grangier, and G. Roger, Phys. Rev. Lett. 49, 91 (1982).

[3] Z. Y. Ou, S. F. Pereira, H. J. Kimble, and K. C. Peng, Phys. Rev. Lett. 68, 3663 (1992).

[4] E. Hagley, X. Maître, G. Nogues, C. Wunderlich, M. Brune, J. M. Raimond, and S. Haroche, Phys. Rev. Lett. 79, 1 (1997).

[5] M. A. Rowe, D. Kielpinski, V. Meyer, C. A. Sackett, W. M. Itano, C. Monroe, and D. J. Wineland, Nature 409, 791 (2001).

[6] B. B. Blinov, D. L. Moehring, L.- M. Duan, and C. Monroe, Nature 428, 153 (2004).

[7] R. Horodecki, P. Horodecki, M. Horodecki, and K. Horodecki, Rev. Mod. Phys. 81, 865 (2009).

[8] H. M. Wiseman, S. J. Jones, and A. C. Doherty, Phys. Rev. Lett. 98, 140402 (2007).

[9] E. G. Cavalcanti, S. J. Jones, H. M. Wiseman, and M. D. Reid, Phys. Rev. A 80, 032112 (2009).

[10] D. J. Saunders, S. J. Jones, H. M. Wiseman, and G. J. Pryde, Nat. Phys. 6, 845 (2010).

[11] E. Schrödinger, Proc. Cambridge Philos. Soc. 31, 553 (1935); 32, 446 (1936).

[12] M. D. Reid, Phys. Rev. A 40, 913 (1989).

[13] E. G. Cavalcanti and M. D. Reid, J. Mod. Opt. 54, 2373 (2007).

[14] E. G. Cavalcanti, M. D. Reid, P. D. Drummond, and H. A. Bachor, Opt. Express 17, 18693 (2009).

[15] Y. Zhang, H. Wang, X. Li, J. Jing, C. Xie, and K. Peng, Phys. Rev. A 62, 023813 (2000).

[16] C. Schori, J. L. Sorensen, and E. S. Polzik, Phys. Rev. A 66, 033802 (2002)

[17] W. P. Bowen, R. Schnabel, P. K. Lam, and T. C. Ralph, Phys. Rev. Lett. 90, 043601 (2003).

[18] J. Laurat, T. Coudreau, G. Keller, N. Treps, and C. Fabre, Phys. Rev. A 70, 042315 (2004).

[19] H. Yonezawa, S. L. Braunstein, and A. Furusawa, Phys. Rev. Lett. 99, 110503 (2007).
[20] G. Keller, V. D’Auria, N. Treps, T. Coudreau, J. Laurat, and C. Fabre, Opt. Express 16, 9351 (2008).

[21] V. D’Auria, S. Fornaro, A. Porzio, S. Solimeno, S. Olivares, and M. G. A. Paris, Phys. Rev. Lett. 102, 020502 (2009).

[22] Y. Wang, H. Shen, X. Jin, X. Su, C. Xie, and K. Peng, Opt. Express 18, 6149 (2010)

[23] T. Eberle, V. Händchen, J. Duhme, T. Franz, R. F. Werner, and R. Schnabel, Phys. Rev. A 83, 052329 (2011).

[24] J. C. Howell, R. S. Bennink, S. J. Bentley, and R. W. Boyd, Phys. Rev. Lett. 92, 210403 (2004).

[25] M. D. Reid, P. D. Drummond, E. G. Cavalcanti, P. K. Lam, H.-A. Bachor, U. L. Andersen, and G. Leuchs, Rev. Mod. Phys. 81, 1727 (2009)

[26] A. J. Bennet, D. A. Evans, D. J. Saunders, C. Branciard, E. G. Cavalcanti, H. M. Wiseman, and G. J. Pryde, Phys. Rev. X 2, 031003 (2012).

[27] B. Wittmann, S. Ramelow, F. Steinlechner, N. K. Langford, N. Brunner, H. Wiseman, R. Ursin, and A. Zeilinger, New J. Phys. 14, 053030 (2012).

[28] D. H. Smith, G. Gillett, M. de Almeida, C. Branciard, A. Fedrizzi, T. J. Weinhold, A. Lita, B. Calkins, T. Gerrits, S. Woo Nam, and A. G. White, Nat. Commun. 3, 625 (2012).

[29] V. Händchen, T. Eberle, S. Steinlechner, A. Samblowski, T. Franz, R. F. Werner, and R. Schnabel, Nat. Photon 6, 596 (2012).

[30] S. L. Braunstein and P. van Loock, Rev. Mod. Phys. 77, 513 (2005).

[31] F. Furrer, T. Franz, M. Berta, A. Leverrier, V. B. Scholz, M. Tomamichel, and R. F. Werner, Phys. Rev. Lett. 109, 100502 (2012).

[32] W. Wasilewski, K. Jensen, H. Krauter, J. J. Renema, M. V. Balabas, and E. S. Polzik, Phys. Rev. Lett. 104, 133601 (2010).

[33] S. Steinlechner, J. Bauchrowitz, M. Meinders, H. MüllerEbhardt, K. Danzmann, and R. Schnabel, arXiv:1211.3570 (2012).

[34] M. Tsang and C. Caves, Phys. Rev. X 2, 031016 (2012). 
[35] M. G. Genoni, M. G. A. Paris, G. Adesso, H. Nha, P. L. Knight, and M. S. Kim, Phys. Rev. A 87, 012107 (2013).

[36] T. Eberle, S. Steinlechner, J. Bauchrowitz, V. Händchen, H. Vahlbruch, M. Mehmet, H. Müller-Ebhardt, and R. Schnabel, Phys. Rev. Lett. 104, 251102 (2010).

[37] M. Mehmet, S. Ast, T. Eberle, S. Steinlechner, H. Vahlbruch, and R. Schnabel, Opt. Express 19, 25763 (2011).
[38] L. M. Duan, G. Giedke, J. I. Cirac, and P. Zoller, Phys. Rev. Lett. 84, 2722 (2000).

[39] V. D'Auria, A. Porzio, S. Solimeno, S. Olivares, and M. G. A. Paris, J. Opt. B 7, S750 (2005).

[40] J. DiGuglielmo, B. Hage, A. Franzen, J. Fiuràšek, and R. Schnabel, Phys. Rev. A 76, 012323 (2007) 